Марданов М.Джс. ${ }^{1}$, Рзаев P.P. ${ }^{2}$

${ }^{1}$ Институт Математики и Механики НАНА, Баку, Азербайджан

${ }^{2}$ Институт Систем Управления НАНА, Баку, Азербайджан

1misir.mardanov@imm.az, ${ }^{2}$ raminrza@yahoo.com

\title{
НЕЧЕТКАЯ ЛОГИКА Л.А.ЗАДЕ КАК КЛЮЧ К ОПИСАНИЮ ГУМАНИСТИЧЕСКИХ СИСТЕМ
}

\begin{abstract}
Прочесс принятия решений является наиболее признанным видом интеллектуальной деятельности человека, который представляется как непрерывная иепь многокритериальных выборов, вырабатываемых и реализуемых в условиях воздействия многочисленных факторов разнообразной природы. $B$ статье с учетом наличия неопределенностей человеческого фактора каждый университет рассматривается как гуманистическая образовательная система, а оченка уровня его конкурентоспособности и вычисление соответствующего рейтинга представляются как слабо структурированная проблема, которая имеет не только количественные, но и качественные характеристики.
\end{abstract}

Ключевые слова: гуманистическая система, лингвистическая переменная, нечеткое множество, нечеткая логика.

\section{Введение}

Ученые, тесно сотрудничавшие с Лотфи Аскер Заде на стыке двух веков, называли его Эйнштейном современности. Многие из них утверждали, что в своих изысканиях он существенно обогатил знания и продвинул научную мысль, воистину совершив в XX веке революцию во взглядах специалистов из самых разных областей на окружающую действительность, и в первую очередь на устоявшиеся нормы классической науки. «Нечеткая логика» Л.Заде стала основой для зарождения многих современных дисциплин, таких как «Нечеткая математика», «Нечеткая физика», «Нечеткая химия», «Нечеткая психология», «Нечеткая топография» и т.д. Обычному человеку и даже некоторым специалистам, не знакомым с работами по приложениям нечеткой логики, трудно понять, в чем состоит суть совершенных Л.Заде открытий. Тем не менее, следует отметить, что Л.Заде - это ученый, на труды которого сейчас в мире ссылаются наибольшее число исследователей. Например, только в последнем десятилетии XX века количество ссылок на его работы составило свыше 36 тысяч.

Нестандартные идеи Л.Заде относительно значимости и роли лингвистических переменных в принятии приближенных решений отразили его новые подходы к описанию слабо структурированных гуманистических систем (подразумевающих участие человека), предоставляя новые возможности для их анализа и решения задач в парадигме здравых рассуждений человека. В частности, в статье [1] И.Б.Турксен (Türkşen I.В.) отмечал: «... важнейший тезис Л.Заде состоит в том, что в гуманистических системах рассуждения и принятие решений основаны не столько на измерениях (хотя они, конечно, являются существенным компонентом), сколько на лингвистических или перцептивных оценках». Благодаря инструментам нечеткой логики удается формализовать всевозможные экспертные вербальные модели, импликативно отражающие причинно-следственные связи между входными и выходными лингвистическими характеристиками. В результате наряду с количественными (метризуемыми) данными стало возможным вовлечь в вычислительный процесс и качественные (неметризуемые, слабо структурированные) категории. 


\section{Этапы на пути становления методов описания гуманистических систем}

Стремительно подняться на Олимп всемирной научной славы Л.А.Заде позволили его основополагающие статьи по нечетким множествам и нечеткой логике, опубликованные в ведущих научных мировых периодических изданиях: «Нечеткие множества» (1965) [2], «Меры вероятности нечетких событий» (1968) [3], «Основы нового подхода к анализу сложных систем и процессов принятия решений» (1973) [4], «Нечеткие множества как основа теории возможности» (1978) [5], «Теория приближенных рассуждений» (1979) [6] и др. Осуществив заметный сдвиг научных парадигм во многих областях, эти работы оказали существенное влияние на развитие классической математики, а также придали заметный импульс развитию естественных, в частности инженерных, и неестественных (гуманитарных) наук. Приведенные статьи привели к многочисленным прикладным открытиям, появлению новых решений в области электроники и зародили основу так называемой Soft Computing технологии, разрабатывающей эффективные гибридные нейронечеткие системы моделирования.

Как же на самом деле выглядит научное и мировоззренческое наследие Л.Заде и в чем оно выражается? Постараемся относительно кратко ответить на этот вопрос.

В 1965 году в своей первой работе [2] Л.Заде вводит общее представление о нечетких множествах как «континуумах степеней принадлежности», определив при этом базовые отношения и операции над ними (равенство, вложенность, дополнение, объединение, пересечение), а также такие понятия, как выпуклая комбинация нечетких множеств; нечеткие множества, индуцируемые отображениями; сепарабельность выпуклых нечетких множеств. Чтобы правильно понять глубинные основы (смысл) теории нечетких множеств, в первую очередь необходимо обратиться к этимологическим корням термина «fuzzy». В статье P.Ходжа (Hodge R.) [7] отмечается, что «нечеткая логика родилась на основе тонкого восприятия Л.Заде различных логик, присущих человеческим языкам.., его интереса к исследованиям силь и слабости естественных языков в научном мышлении». Обладая большими способностями в области языкознания, Л.Заде применяет термин «fuzzy» как категорию языка или мышления, что, по сути, не имеет отношения к природе физических, механистических явлений. Однако к концу XX века Л.Заде в статье [8] предложил так называемую типологию граничных или краевых условий протекания физических явлений в терминах описывающих их категорий, где он, в частности, описывает то, как люди воспринимают и идентифицируют друг друга по чертам лица.

Начальная фаза в развитии нечеткой логики основывалась на доказательстве Л.Заде относительно необходимости ослабления логического закона, исключающего третьего, и двойственного ему закона логики о непротиворечивости. Со времен Аристотеля эти законы считались незыблемыми в классической теории множеств. Новейшая «поствиннеровская» история показала, что для открытия и разработки новых теорий надо отбросить традиционный образ мышления. В результате научный мир разделился на две части: приверженцев классических подходов к достижению искусственного интеллекта («нисходящий путь») и приверженцев «восходящего» пути, отстаивающих создание нового мышления и новых подходов. Собственно, отвергнув главную аксиому классической теории, Л.Заде осуществил в 1965 году этот рывок по восходящему пути развития.

Тремя годами позже, в 1968 году, в статье [3], посвященной мерам вероятности нечетких событий, Л.Заде ввел понятие «нечеткое явление», которое иллюстрируется на примерах, таких как «сегодня ясный день», «значение $x$ около 7», «при тестировании на наличие вируса получено чуть больше отрицательных, чем положительных результатов». Из-за неточности смысла составляющих слов эти выражения являются слабо структурированными, т.е. такими, о которых известна их принадлежность к определенному типу. Именно такие выражения Л.Заде считал нечеткими обобщениями математических 
выражений, по средствам которых он обобщил понятия среднего арифметического, вариации, энтропии на случай нечетких событий.

Как известно, основу теории нечетких множеств 1-го типа (Fuzzy-1) составляют функции принадлежности, которые хорошо описывают отчетливо выраженные границы. Однако когда границы выражены некоторыми зонами, т.е. плохо различимы (размыты), то их Л.Заде выражает по средствам нечетких множеств 2-го типа (или Fuzzy-2) и далее по порядку. В частности, нечеткое множество 2 -го типа $\tilde{A}$ определяется как

$$
\tilde{A}=\left\{\left((u, i), \mu_{\tilde{A}}(u, i)\right) \mid \forall u \in U \text { и } \forall i \in J_{u} \subseteq[0,1]\right\},
$$

где $U$ - универсум; $\mu \tilde{A}(u, i)$ - соответствующая функция принадлежности 2-го типа, удовлетворяющая условию $0 \leq \mu \tilde{A}(u, i) \leq 1$. Если универсум $U$ является континуумом, то его нечеткое подмножество выражается в следующем виде:

$$
\tilde{A}=\int_{u \in U} \int_{i \in J_{u}} \mu_{\tilde{A}}(u, i) /(u, i), J_{u} \subseteq[0,1],
$$

где символ « $\int_{\gg}$ отражает объединение по всем допустимым $u$ и $i$. В случае дискретного

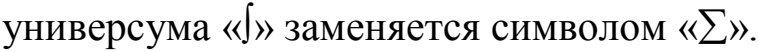

В случаe Fuzzy-2 отдельные значения принадлежности элементов к нечетким множествам задаются функциями принадлежности 2-го типа, т.е. в этом случае учитывается сама неточность в определении принадлежности. Такие функции принадлежности могут выражать лингвистическую неопределенность, связанную с различными семантическими оттенками тех или иных слов (рис. 1б). В практике общения многие слова имеют неточные смысловые значения, которые даже в случае одного контекста порой интерпретируются как двусмысленность и/или размытость. Например, по средствам нечетких множеств 2-го и выше типов можно отражать величины рисков, имеющих место в процессе принятия решений в самых разных областях.

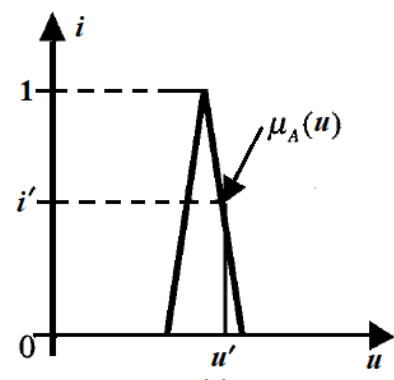

(a)

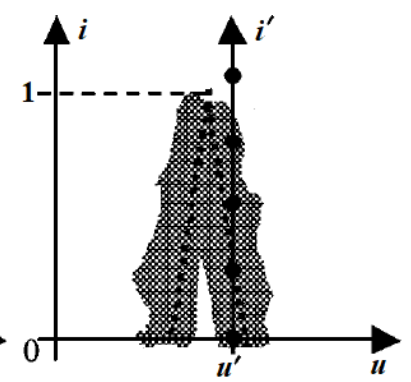

(ס)

Рис. 1. $A$ - треугольная функция принадлежности 1-го типа; $\sigma$ - «размытая» функция принадлежности 1-го типа, включающая дискретизацию при $u=u^{\prime}$.

Следующий важный шаг в развитии и применении нечеткой логики нашел свое отражение в [9], посвященной принятию решений в нечеткой среде, которая была опубликована в 1970 году Л.Заде в соавторстве с Р.Е.Беллманом (Bellman R.E.). B частности, в статье на примерах многошаговых процессов принятия решений иллюстрируется принципиально новый подход, в котором ключевые цели и/или базовые ограничения задаются в слабо структурированном виде, т.е. имеют нечеткую природу. Впоследствии этот подход стали называть принципом Беллмана-Заде в принятии решений в условиях неопределенности или, более конкретно, в решении задачи многокритериальной оптимизации при наличии нечеткой информации. Сама задача формулируется следующим образом:

$$
\min _{\dot{x}}\left\{f_{1}(x), f_{2}(x), \ldots, f_{k}(x)\right\}, \rho=\left(x_{1}, x_{2}, \ldots, x_{n}\right)^{T} \in S,
$$


где $f_{k}: R^{n} \rightarrow R(k \geq 2)$ - целевые функции, описывающие критерии удовлетворительности и, как правило, взаимно противоречащие друг другу; $S$ - непустая область определения.

Принятие решений в нечеткой среде по принципу Беллмана-Заде - это многокритериальный выбор наилучшей альтернативы, которая одновременно удовлетворяет нечетким целям (качественным критериям) и нечетким ограничениям (рис. 2). В данном случае между целью и ограничениями не делается никаких различий. Если есть такое различие, то оно носит условный характер.

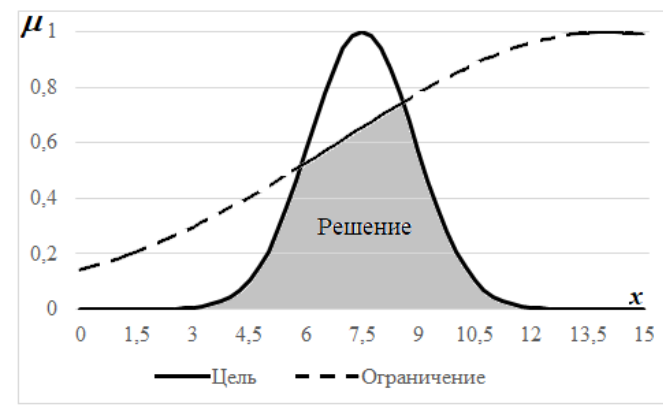

Рис. 2. Иллюстрация принятия решений по принципу Беллмана-Заде

В 1971 году появилась новая статья Л.Заде [10] об отношениях подобия и нечетких порядках. В этой статье Л.Заде рассматривает отношение подобия как «нечеткое отношение», обладающее свойствами рефлексивности, симметричности и транзитивности, а также нечеткие линейные порядки, нечеткие предпорядки и слабые порядки. По сути, эта работа подвела черту под пятилетним исследованием Л.Заде, когда он разработал основы теории нечетких множеств как предпосылки для последующих практических приложений.

С каждым годом процесс принятия решений и технологии управления сталкивается с постоянно усложняющимися условиями, где одним из главных факторов неопределенности является экспоненциальный рост сложности информационной ситуации, в которой находится объект управления. Другим отягощающим фактором является возрастание сложности системы управления из-за сложности, собственно, самого объекта управления, характеризуемого распределенным характером и наличием многочисленных связей, включая и нестационарные. Поэтому все это диктует необходимость применения новых подходов и моделей сложных систем в технологии управления.

В 1973 году Л.Заде впервые вводит понятие лингвистической переменной и композиционное правило логического вывода [4], что в скором времени находит свое отражение в развитии систем нечеткого вывода. В статье подчеркивается, что ограниченное число лингвистических правил способно обеспечить хоть и приблизительное, но все же эффективное средство описания поведения слабо структурированных систем, которые являются плохо формализуемыми для того, чтобы применять к ним точные математические методы. Слабо структурированные системы (задачи) чаще всего встречаются в экономике, менеджменте, информационном поиске, искусственном интеллекте, психологии, лингвистике, медицине, биологии и т.д., т.е. в областях, где присутствует человеческий фактор. В продолжении этой работы в 1975 году Э.Мамдани (Mamdani Е.) и С.Ассилиан (Assilian S.) [11] предложили систему лингвистических правил в качестве нечеткого регулятора, применили ее в промышленности и тем самым заложили основу промышленного нечеткого управления.

В качестве ключевой парадигмы дальнейшего развития теории нечетких множеств следует особо выделить суждение Л.Заде: «Высокие стандарты точности, которые преобладают в математике, физике, химии, инженерных науках и т.n., находятся в явном противоречии с неточностью наших знаний, которая типична для сочиологии, психологии, 
лингвистики, истории, философии, антропологии, литературы, искусства и других областей», которое он сформулировал в 1976 году в статье «Нечетко-алгоритмический подход к определению сложных или неточных понятий». Другой важной парадигмой можно считать утверждение Л.Заде: «Когда наш основной интерес cocmoum скорее в описании смысла информации, чем в определении ее меры (в смысле работ Шеннона и Винера по статистической теории информации), возможностный подход $\kappa$ анализу информации оказывается более удобным и естественным по своей природе, чем вероятностный», которое он привел в статье «Нечеткие множества как основа теории возможности», опубликованной в 1978 году В этой же статье Л.Заде вводит в оборот понятия «распределение возможностей», «меры возможности», «качественное описание истинности», «вероятностная квалификация» и пр.

Важнейшим этапом на пути развития нечеткой логики стали статьи Л.Заде под общим названием «Понятие лингвистической переменной и его применение к принятию приближенных решений» [12, 13], опубликованные в 1975 и 1976 годах. Эту серию открывает утверждение автора: «Под лингвистической переменной понимается такая переменная, значениями которой являются слова и словосочетания на некотором естественном или искусственном языке». Там же Л.Заде отмечает: «С учетом нашего преклонения перед всем точным, строгим и количественным и нашего пренебрежения ко всему нечеткому, нестрогому и качественному неудивительным кажется приход цифровых компьютеров, которые оказались весьма эффективными при работе с механистическими, т.е. неживыми системами, поведение которых определяется законами механики, физики, химии, электромагнетизма. К сожалению, этого нельзя сказать о гуманистических системах...»

В своей следующей работе «Теория приближенных рассуждений» [6], опубликованной в 1979 году, Л.Заде детально анализирует возможности системы нечеткого вывода с точки зрения обеспечения приближенных рассуждений с учетом принципов проекции, логического следования семантической эквивалентности и т.д.

Научные труды профессора Л.Заде оказали огромное влияние на развитие теории искусственного интеллекта. Так, в статье «Роль нечеткой логики в управлении неопределенностью в экспертных системах», опубликованной в 1983 году, Л.Заде подчеркивает: «Задача управления неопределенностью есть жизненно важный вопрос проектирования экспертных систем, поскольку большая часть информации в базе знаний типичной экспертной системы является неточной, неполной или не совсем надежной».

В серии работ под общим названием «Нечеткая логика - вычисления со словами» $[14$, 15], опубликованных в период с 1996 по 2001 год, Л.Заде утверждает: «Основной вклад нечеткой логики заключается в построении методологии для вычислений со словами. Для этого пока нет никакой другой методологии». Там же профессор делает очень важное замечание: «Вычисления в обычном смысле включают манипулящии числами и символами. Напротив, люди в основном пользуются словами при счете и рассуждениях, приходя к словесным заключениям из посылок, выраженных средствами натурального языка или выступаюших в форме психических (перчептивных) образов».

В заключение приведем основные мысли профессора Л.Заде, которые можно считать лейтмотивами для последующих исследований в области искусственного интеллекта:

- «B наших поисках оснований для машинного интеллекта (с высоким коэффициентом интеллектуальности MIQ) мы пришли к лучшему пониманию фундаментального значения одной из самых замечательных человеческих способностей. Это - способность выполнять широкий круг физических и психических задач без каких-либо измерений или вычислений. В основе этой способности лежит важнейшее свойство человеческого мозга, связанное с манипулированием сенсорно-перцептивными образами: ощущениями расстояния, 
размера, веса, силы, цвета, сходства, истинности и других физических и психических характеристик».

- Основное отличие между перцептивными оценками и измерениями заключается, прежде всего, в том, что измерения являются четкими, тогда как оценки нечеткими».

- «Люди пользуются словами для описания своих перцептивных образов, и в этом плане манипулирование перцептивными образами сводится к вычислению со словами...»

- «В ближайшие годы вычисления со словами и перцептивными оценками, повидимому, станут одним из важнейших направлений в науке и технике».

Всю жизнь проработавший в крупнейших университетах США, профессор Л.А.Заде одновременно является действительным и почетным членом академий многих зарубежных стран. Этого же звания его удостоила и Национальная Академия Наук Азербайджана в 1965 году, когда он впервые после тридцатилетнего отсутствия посетил Баку - город, где родился и где прошло его детство.

\section{Применение нечеткой логики для оценки конкурентоспособности университетов и вычисления их рейтингов}

В Азербайджане достаточно много специалистов из разных областей, которые активно применяют достижения Л.Заде для решения тех или иных прикладных задач. В частности, нами на протяжении последних пяти лет была разработана методологическая основа системы информационно-аналитической поддержки для оценки конкурентоспособности университетов и вычисления их рейтингов. Отправным здесь было то, что сама задача является слабо структурированной, а сам университет представляется как гуманистическая система. Л.А.Заде всегда выступал против традиции, укрепляющей растущую тенденцию анализировать гуманистические системы так, как если бы они были механистическими системами, описываемыми в терминах разностных, дифференциальных или интегральных уравнений. В свое время он писал: «Наш основной тезис заключается 8 том, что по своей сути обычные количественные методы анализа систем непригодны для гуманистических систем и вообще любых систем, сравнимых по сложности с гуманистическими системами... Чем сложнее система, тем менее мы способны дать точные и в то же время имеющие практическое значение суждения о ее поведении». В частности, Л.А.Заде считал, что точный количественный анализ не имеет, по-видимому, большого практического значения для решения реальных социальных, экономических и других задач, обусловленных активным участием человека.

Комплексная оценка конкурентоспособности университетов, как слабо структурированная задача, требовала мультидисциплинарного подхода. Поэтому для ее решения нами были использованы методы и модели математической статистики, элементы нечеткой логики, теорий нечетких множеств и нейронных сетей, а также гибридные (нейронечеткие) системы и современные информационные технологии. По результатам проведенных исследований были опубликованы девять статей и одна монография.

В 2015 году нами были предложены модели для оценки маркетингового пространства образовательных услуг и конкурентной позиции университета на рынке труда, разработанные на основе применения метода нечеткого логического вывода [16]. В контексте данного исследования предложен нечеткий подход к формированию гибкой и универсальной системы оценки конкурентоспособности университетов. В том же году в ведущем российском журнале «Проблемы управления» (Москва) была опубликована статья [17], где нами был предложен метод для оценки конкурентоспособности университетов с последующим их ранжированием, основанный на применении системы нечеткого логического вывода. Здесь анализ конкурентоспособности университетов включает 
многокритериальные оценки маркетинговой среды и конкурентной позиции университетов на рынке образовательных услуг. Так, маркетинговая среда университетов оценивалась по 13 показателям: $x_{1}$ - поддержка государства; $x_{2}$ - конкурс абитуриентов; $x_{3}-$ связь со средними школами; $x_{4}$ - популяризация медийными структурами; $x_{5}-$ состояние национальной экономики; $x_{6}$ - социально-демографическая ситуация; $x_{7}$ - социальнокультурное положение общества; $x_{8}$ - политико-правовое обеспечение общества; $x_{9}$ целевое ориентирование; $x_{10}$ - применяемая технология обучения; $x_{11}$ - организационная структура университета; $x_{12}$ - уровень профессорско-преподавательского состава; $x_{13}-$ число вузов-конкурентов; а оценка конкурентных позиций специальностей университетов - по двум показателям: $y_{1}$ - рыночная доля университета по данной специальности; $y_{2}-$ темп прироста рыночной доли университета по данной специальности. В обоих случаях для описания качественных критериев оценки (термов лингвистических переменных $x_{i}(i=1 \div 13)$ и $\left.y_{j}(j=1,2)\right)$ по средствам подходящих нечетких множеств $\tilde{A}$ использовалась Гауссовская функция принадлежности вида:

$$
\mu_{\tilde{A}}(u)=\exp \left[-\left(u-u_{0}\right)^{2} / \sigma^{2}\right],
$$

где $u$ - показатель университета по текущей позиции; $u_{0}$ - среднее значение показателей всех университетов по текущей позиции; $\sigma^{2}$ - плотность (дисперсия).

Оценочные суждения относительно маркетинговой среды и конкурентной позиции университетов строились в виде термов выходных лингвистических переменных, описываемых соответствующими нечеткими подмножествами дискретного универсума $J=\{0,0.1,0.2, \ldots, 1\}$. В частности, $\forall j \in J$ такими термами являются: $F=$ БЛАГОПРИЯТНАЯ, $\mu_{F}(j)$ $=j ; M F=$ БОЛЕЕ ЧЕМ БЛАГОПРИЯТНАЯ, $\mu_{M F}(j)=\sqrt{j} ; V F=$ ОЧЕНЬ БЛАГОПРИЯТНАЯ, $\mu_{V F}(j)=j^{2}$; $P=$ БЕЗУПРЕЧНАЯ, $\mu_{P}(j)=\left\{\begin{array}{l}1, \text { если } j=1, \\ 0, \text { если } j<1 ;\end{array} U F=\right.$ НЕБЛАГОПРИЯТНАЯ, $\mu_{U F}(j)=1-j$.

Апробация подхода в [17] проведена на примере пяти гипотетических университетов посредством системы нечеткого вывода. В результате общее функциональное решение, например, относительно оценки маркетинговой среды университетов, выглядит в виде матрицы

$$
R=\left[\begin{array}{l|ccccccccccc} 
& 0 & 0.1 & 0.2 & 0.3 & 0.4 & 0.5 & 0.6 & 0.7 & 0.8 & 0.9 & 1 \\
\hline u_{1} & 0.8701 & 0.9701 & 0.9870 & 0.9870 & 0.9679 & 0.8679 & 0.7679 & 0.6679 & 0.5679 & 0.4679 & 0.3679 \\
u_{2} & 0.3874 & 0.4874 & 0.5874 & 0.6874 & 0.7874 & 0.7904 & 0.7904 & 0.7904 & 0.7904 & 0.7904 & 1.0000 \\
u_{3} & 0.9588 & 0.9817 & 0.9817 & 0.9441 & 0.8441 & 0.7441 & 0.6441 & 0.5441 & 0.4441 & 0.3441 & 0.2441 \\
u_{4} & 0.6827 & 0.7827 & 0.8827 & 0.9827 & 0.9870 & 0.9870 & 0.8894 & 0.7994 & 0.6994 & 0.5994 & 0.4994 \\
u_{5} & 0.8946 & 0.9777 & 0.9777 & 0.9777 & 0.9777 & 0.9777 & 0.9777 & 0.9777 & 0.9777 & 0.8788 & 0.7788
\end{array}\right],
$$

где каждая строка - суть значений функции принадлежности нечеткого подмножества дискретного универсума $J: \mu_{E i}(j), i=1 \div 5 ; j=0,0.1,0.2, \ldots, 1$, отражающего нечеткий вывод относительно оценки маркетингового пространства соответствующего университета.

Для дефаззификации нечетких оценочных выводов $E_{i}$ применялась формула:

$$
F\left(E_{i}\right)=\frac{1}{\alpha_{\max }} \int_{0}^{\alpha_{\max }} M\left(E_{i \alpha}\right) d \alpha, i=1 \div 5,
$$

где $E_{i \alpha}-\alpha$-уровневое множество $(\alpha \in[0,1])$, определяемое в виде $E_{\alpha}=\left\{j \mid \mu_{E i}(i) \geq \alpha, j \in I\right\} ; \alpha_{\max }$ - максимальное значение на $E_{i \alpha} ; M\left(E_{i \alpha}\right)=\frac{1}{n} \sum_{k=1}^{n} x_{k}$ - мощность множества $E_{i \alpha}$.

В 2016 году в [18] нами был предложен альтернативный подход к агрегации оценок ключевых показателей университетов по версии QS, основанный на применении метода 
нечеткого логического вывода. В качестве оцениваемых альтернатив были выбраны университеты мира, занявшие по версии QS WUR первые десять мест из списка. По результатам проведенных расчетов получено ранжирование топ-университетов, которое несколько отличается от версии QS WUR, основанной на взвешенной агрегированной оценке ключевых показателей.

Годом позже для оценки и последующего ранжирования университетов на предмет качества предоставляемых ими образовательных услуг нами были применены два подхода: статистический, основанный на взвешенных оценках ключевых показателей университетов, и вербальныц̆, основанный на применении механизма нечеткого логического вывода $[19,20]$. По результатам применения данных методов к оценке произвольно выбранных университетов (гипотетических альтернатив) были получены схожие агрегированные результаты (рейтинги) и соответствующие им два способа ранжирования. Было отмечено, что в случае охвата большего количества университетов, например, как и в случае QS WUR методики, качество фаззификации критериев оценки показателей заметно улучшится и, как следствие, повысится точность искомых оценок.

В 2017 году в немецком издательстве «Palmarium Academic Publishing» нами была опубликована книга «Нечеткая методология многокритериального ранжирования университетов» [21], в которой излагаются нечеткие методы многокритериального выбора альтернатив в условиях неопределенности, а также новые подходы к решению задач, связанных с всеобъемлющей оценкой образовательных услуг, ранжированием университетов и оптимизацией их организационных структур.

В 2018 и 2019 годах нами были рассмотрены нечеткие методы многокритериальной оценки альтернатив в условиях недостаточности информации [22, 23]. С целью аналитической поддержки процесса принятия решений сформулированы новые подходы к компиляции приобретенных знаний путем адекватного перевода внешнего представления знаний о взвешенных суммарных оценках альтернатив, полученных на основе экспертных заключений, в эффективные внутренние представления в логическом базисе многослойной feedforward нейронной сети.

Наконец, в 2019 году в статье [24] нами была предложена методика конструктивной оценки эффективности деятельности университетов с привлечением множества критериев, скорректированных под региональную специфику. Данное множество состоит из 18 критериев и разбито на три раздела - по ключевым показателям «Потенциал», «Активность и качество образования», «Международное признание». Некоторые из них вычисляются как доли объектов или результатов деятельности университета определенного качества относительно общего их числа или объема. Другие показатели носят исключительно качественный характер, например, такие как привлекательность среди абитуриентов и/или репутация среди работодателей. Ряд показателей являются расчетными по тем или иным схемам, как, например, уровень участия университета в международных программах. Остальные представляют собой абсолютные значения измерений. Предлагаемая методика учитывает тот факт, что различные показатели деятельности университетов имеют разные веса в определении их рейтинга, а их приоритеты должны меняться в зависимости от специфики университета, его размера, возраста и ряда других характеристик. В результате подобного анализа производится не только ранжирование университета, но и намечается механизм его поступательного развития. Исходя из этих соображений, в рамках указанной методики предлагается подход к многокритериальной оценке образовательных услуг и ранжированию университетов, основанный на комбинированном применении экспертных оценок и нейросетевого моделирования.

Предварительный экспертный анализ относительного влияния факторов, отражающих качество образовательных услуг, включал: 1) отбор факторов влияния; 2) идентификацию обобщенных весов для отобранных факторов, исходя из их 
относительного влияния; 3) формирование ключевых показателей образовательных услуг и, соответственно, взвешенных суммарных индексов - рейтингов университетов.

Для установления ранговых оценок факторов $x_{k i}(i=1 \div n)$ привлекаются эксперты, которым предлагается ранжировать переменные $x_{k i}$ по следующему принципу: наиболее важную индексировать цифрой «1», следующую, менее важную, - цифрой «2» и далее по убыванию в порядках предпочтения эксперта. Полученные таким образом ранговые оценки суммируются и оцениваются на согласованность. Для определения степени согласованности экспертных заключений относительно приоритетности $x_{k i}(i=1 \div n)$ применяется коэффициент конкордации Кендалла, демонстрирующий множественную ранговую корреляцию экспертных мнений:

$$
W=\frac{12 \cdot S}{m^{2}\left(n^{3}-n\right)},
$$

где $m$ - число привлеченных экспертов; $n$ - число факторов; $S$ - отклонение экспертных заключений от среднего значения ранжирования ФВ $x_{i}(i=1 \div n)$, которое вычисляется как:

$$
S=\sum_{i=1}^{n}\left(\sum_{j=1}^{m} r_{i j}-\frac{m(n+1)}{2}\right)^{2},
$$

где $r_{i j} \in\{1,2, \ldots, n\}$ - ранг $i$-го фактора влияния, установленный $j$-ым экспертом.

На предварительном этапе независимого анкетирования каждому из приглашенных экспертов также предлагается установить значения нормированных оценок обобщенных весов $x_{k i}(i=1 \div n)$ в виде $\alpha_{i j}$. Далее рассчитываются групповые оценки факторов влияния и степени компетентности экспертов. Чтобы вычислить среднюю величину $\alpha_{i}$ по $i$-ой группе нормированных оценок обобщенных весов $x_{k i}$, применяется равенство:

$$
\alpha_{i}(t+1)=\sum_{j=1}^{m} w_{j}(t) \alpha_{i j}
$$

где $w_{j}(t)$ - коэффициент, характеризующий степень компетентности $j$-го эксперта $(j=1 \div m)$ на момент времени $t$. Как видно из (4), процесс нахождения групповых оценок нормированных значений обобщенных весов ФВ является итерационным и завершается при выполнении условия: $\max _{i}\left\{\left|\alpha_{i}(t+1)-\alpha_{i}(t)\right|\right\} \leq \varepsilon$, где $\varepsilon$ - допустимая точность расчетов.

Показатели компетентности экспертов $w_{j}(t)(j=1 \div m)$ на момент времени $t$ вычисляются на основании следующих равенств:

$$
\left\{\begin{array}{l}
w_{j}(t)=\frac{1}{\eta(t)} \sum_{i=1}^{n} \alpha_{i}(t) \cdot \alpha_{i j}(j=\overline{1, m-1}), \\
w_{m}(t)=1-\sum_{j=1}^{m-1} w_{j}(t), \quad \sum_{j=1}^{m} w_{j}(t)=1 .
\end{array},\right.
$$

где $w_{j}(t)$ - показатель компетентности $j$-го эксперта в $t$-ом приближении, а $\eta(t)-$ нормирующий множитель, вычисляемый по формуле: $\eta(t)=\sum_{i=1}^{n} \sum_{j=1}^{m} \alpha_{i}(t) \alpha_{i j}$.

Полученные на основе экспертных заключений знания, как внешние представления о взвешенных суммарных оценках университетов, компилируются в эффективные внутренние представления о них в логическом базисе трехслойной feedforward нейронной сети, индуцирующие на своих выходах сигналы вида: 


$$
z_{j}=\sum_{p=1}^{m} c_{p} \varphi\left[\sum_{i=1}^{r} w_{p i} x_{i j}-\theta_{p}\right](j=1 \div 50)
$$

где $r$ - число компонентов входного вектора; $m$ - число нелинейных нейронов в «скрытом» слое, подбираемое пользователем в процессе симуляции методом проб и ошибок; $w_{p i}$ и $c_{p}-$ веса входных и выходных синоптических связей соответственно; $\theta_{p}$ - порог (смещение) $p$ го нелинейного нейрона из «скрытого» слоя; $\varphi(\cdot)$ - функция активации нелинейного нейрона из «скрытого» слоя, например, сигмоидального типа $\varphi(t)=1 /\left(1+e^{-t}\right)$.

\section{Заключение}

Главным месседжем Л.Заде, направленным всему научному сообществу, является то, что в гуманистических системах рассуждения и принятие решений основаны не только на измерениях (количественных оценках), но и на лингвистических оценках. Благодаря технологиям нечеткой логики в вычислительный процесс удается вовлечь как количественные, так и качественные характеристики.

Комплексный показатель качества образовательных услуг в университете отражает очень широкий спектр разнородных параметров, характеризующих степень соответствия образовательных программ, материально-техническое обеспечение учебного процесса, научно-методическую базу, кадровый состав и пр. Согласно Дж. фон Нейману, стремление получить адекватную модель для оценки слабо структурированной системы, каковым, несомненно, является образовательный процесс в университете, теряет смысл, поскольку ее сложность становится соизмеримой со сложностью самого процесса. Очевидно, что применение такой модели не может позволить относительно просто и наглядно интерпретировать механизм образовательного процесса, воспользоваться какими-либо формальными процедурами для исследования его характеристик и синтеза системы управления им. Более того, для образовательного процесса, как сложной, открытой и динамически развивающейся системы, применим принцип несовместимости Л.Заде [4], согласно которому невозможно получить точные и в то же время имеющие практическое значение суждения о ее текущем и будущем состояниях. Поэтому многими рейтинговыми агентствами статистический расчет ключевых показателей качества образовательных услуг производился путем взвешенного суммирования их составляющих с заранее выбранными весами, для идентификации которых прибегают к применению экспертных систем, где основным ресурсом выступают эвристические знания профильных специалистов.

Обычно экспертные системы обоснованно критикуют за то, что они не отражают причинно-следственные связи. Аналитический же подход к оценке альтернатив позволяет сравнивать альтернативы посредством индекса, обобщающего в себе относительное влияние конечного числа факторов в виде многофакторной функции вида $F=F\left(x_{1}, x_{2}, \ldots, x_{n}\right)$. Тем не менее, в условиях отсутствия достаточного объема количественных данных, формирующих числовое представление об оцениваемых показателях, информацию о критериях оценки, предпочтениях, применяемые в принятии решений эконометрические модели вида $F$ страдают сложностью обеспечения текущими источниками данных факторов. Поэтому рабочую модель целесообразно представлять в виде «черного ящика», входы и выходы которого определяются экспертными оценками.

\section{Литература}

1. Turksen I.B. O vklade Lotfi Zade v sovremennuyu nauku i nauchnoe mirovozzrenie // Novosti Iskusstvennogo Intellekta, 2001, \# 2-3, s. 12-15. Zadeh L.A. Fuzzy Sets // Information and Control, 1965, Vol.8, p. 338-353.

2. Zadeh L.A. Fuzzy Sets // Information and Control, 1965, Vol.8, p. 338-353. 
3. Zadeh L.A. Probability Measures of Fuzzy Events // Journal of Mathematical Analysis and Applications, 1968, Vol.10, p. 421-427.

4. Zadeh L.A. Outline of a New Approach to the Analysis of Complex Systems and Decision Processes // IEEE Transactions on Systems, Man and Cybernetics, 1973, Vol. SMC-3, p. 28-44.

5. Zadeh L.A. Fuzzy Sets as a Basis for a Theory of Possibility // Fuzzy Sets and Systems, 1978, Vol.1, p. 3-28.

6. Zadeh L.A. A Theory of Approximate Reasoning // Machine Intelligence / Ed. by J. Hayes, D. Michie and L.I. Mikulich, New York: Halstead Press, 1979, p.149-194.

7. Hodge R. Key Terms in Fuzzy Logic Deep Roots and New Understanding, University of Western Sydney, Australia, 2001.

8. Zadeh L.A. Toward a Theory of Fuzzy Information Granulation and its Centrality in Human Reasoning and Fuzzy Logic // Fuzzy Sets and Systems, 1997, Vol. 90, p.111-127.

9. Bellman R.E., Zadeh L.A. Decision-Making in a Fuzzy Environment // Management Science, 1970, Vol.17, p.141-164.

10. Zadeh L.A. Similarity Relations and Fuzzy Orderings // Information Sciences, 1971, Vol.3, p.177-200.

11. Mamdani E.H., Assilian S. An Experiment in Linguistic Synthesis with a Fuzzy Logic Controller // International Journal of Man-Machine Studies, 1975, Vol. 7, №1, p.1-13.

12. Zadeh L.A. The Concept of a Linguistic Variable and its Application to Approximate Reasoning. Parts 1 and 2 // Information Sciences, 1975, Vol.8, p. 199-249, 301-357.

13. Zadeh L.A. The Concept of a Linguistic Variable and its Application to Approximate Reasoning. Part 3 // Information Sciences, 1976, vol. 9, p. 43-80.

14. Zadeh L.A. Fuzzy Logic $=$ Computing with Words // IEEE Transactions on Fuzzy Systems, 1996, Vol. 4, p.103-111.

15. Zadeh L.A. From Computing with Numbers to Computing with Words - From Manipulation of Measurements to Manipulation of Perceptions // Computing with Words/ Ed. by P.P. Wang, New York: Wiley and Sons, 2001, p. 35-68.

16. Mardanov M.Dzh., Rzaev R.R., Dzhamalov Z.R., Khudadova A.K. Oczenka konkurentosposobnosti vy`sshego uchebnogo zavedeniya na osnove nechyotkogo analiza ego kachestvenny`kh kharakteristik // Izvestiya Azerbajdzhanskoj Inzhenernoj Akademii, 2015, tom 7, №4, s. 113-130.

17. Mardanov M.Dzh., Rzaev R.R., Dzhamalov Z.R., Gasanov V.I. Podkhod k oczenke konkurentosposobnosti vy`sshikh uchebny`kh zavedenij // Problemy` upravleniya. Moskva, 2015, №6, s. 23-34.

18. Mardanov M.Dzh., Rzaev R.R. Al ternativnoe agregirovanie rejtingovy`kh oczenok v ramkakh QStekhnologii ranzhirovaniya vy`sshikh uchebny`kh zavedenij // Matematicheskie Mashiny`i Sistemy`. Institut Problem Matematicheskikh Mashin i Sistem, Kiev, 2016, № 4, c. 122-133.

19. Mardanov M.Dzh., Rzaev R.R. Dva podkhoda k kompleksnoj oczenke i ranzhirovaniyu vuzov // Problemy` upravleniya i informatiki, 2017, № 4, c. 36-55.

20. Mardanov M.Dzh., Rzaev R.R., Dzhamalov Z.R., Khudadova A.K. Integrated Assessment and Ranking of Universities by Fuzzy Inference // Procedia Computer Science, 2017, Vol. 120, p. 213-220.

21. Mardanov M.Dzh., Rzaev R.R., Dzhamalov Z.R. Nechyotkaya metodologiya mnogokriterial nogo ranzhirovaniya universitetov, Saarbruchen (Germany): Palmerium Academic Publishing, 2017, 164 p.

22. 21. Mardanov M.Dzh., Rzaev R.R. Metody` mnogokriterial`noj oczenki al’ternativ v logicheskom bazise nejronny`kh setej // Nauchny`j zhurnal «Avtomatizacziya i izmereniya v mashino-priborostroenii», 2018, № 3, s. 75-85. 
23. Mardanov M.Dzh., Rzaev R.R. One Approach to Multi-Criteria Evaluation of Alternatives in the Logical Basis of Neural Networks // Springer's Series “Advances in Intelligent Systems and Computing", 2019, Vol. 896, p. 279-287.

24. Mardanov M.Dzh., Rzaev R.R., Ibragimov N.S., Dzhamalov Z.R. Formirovanie rejtingov universitetov na osnove kompilyaczii e`kspertny`kh oczenok v logicheskom bazise nejronnoj seti // Matematicheskie Mashiny` i Sistemy`. Institut Problem Matematicheskikh Mashin i Sistem, Kiev, 2019, № 1, c. 107-123.

\title{
UOT 338
}

\author{
Mərdanov Misir C. ${ }^{1}$, Rzayev Ramin R. ${ }^{1}$ \\ ${ }^{1}$ AMEA-nın Riyaziyyat və Mexanika İnstitutu, Azərbaycan, Bak1 \\ ${ }^{2}$ AMEA-nın İdarəetmə Sistemləri İnstitutu, Azərbaycan, Bakı \\ ${ }^{1}$ misirmardanov@yahoo.com, ${ }^{2}$ raminrza@yahoo.com
}

\section{Zadənin qeyri-səlis məntiqi humanistik sistemlərin təsviri üçün bir açar kimi}

Çoxsaylı, müxtəlif təbiətli faktorların təsiri altında işlənilən və realizə olunan çox meyarlı seçimlərin kəsilməz zənciri kimi təsvir olunan qərarların qəbul edilməsi prosesi insanın intellektual fəaliyyətinin bir növü kimi qəbul edilir. Məqalədə insan faktorunun, qeyrimüəyyənliklərin mövcudluğu şəraitində hər bir universitetə humanistik təhsil sistemi kimi baxılır. Universitetin rəqabət qabiliyyətlilik səviyyəsinin qiymətləndirilməsi və müvafiq reytinqinin hesablanması isə kəmiyyət və keyfiyyət xassələrilə fərqlənən zəif strukturlaşmış problem kimi təsvir olunur.

Açar sözlor: humanistik sistem, linqvistik dayişən, qeyri-səlis çoxluq, qeyri-səlis məntiq.

\section{Misir J. Mardanov ${ }^{1}$, Ramin R. Rzayev ${ }^{2}$}

${ }^{1}$ Institute of Mathematics and Mechanics of ANAS, Azerbaijan, Baku

${ }^{2}$ Institute of Control Systems of ANAS, Azerbaijan, Baku

${ }^{1}$ misirmardanov@yahoo.com, ${ }^{2}$ raminrza@yahoo.com

\section{L.A.Zadeh's "Fuzzy logic" as a key to the description of humanistic systems}

The decision-making process is recognized as a type of human intellectual activity, which is presented as a continuous chain of multi-criteria choices developed and implemented under the influence of numerous factors of the diverse nature. In the paper, considering the presence of uncertainties, and the human factor, each university is considered as a humanistic educational system, and the assessment of its competitiveness level and the calculation of the corresponding rating are presented as a weakly structured problem that has not only quantitative but also qualitative characteristics.

Keywords: humanistic system, linguistic variable, fuzzy set, fuzzy logic. 